\title{
Optical waveplates based on birefringence of anisotropic two-dimensional layered materials
}

\author{
He Yang, ${ }^{\dagger}$ Henri Jussila, ${ }^{\dagger}$ Anton Autere, ${ }^{\dagger}$ Hannu-Pekka Komsa, ${ }^{\ddagger}$ Guojun Ye, $\|,{ }^{\perp}$ \\ Xianhui Chen, $\|, \perp$, § Tawfique Hasan, ${ }^{\top}$ and Zhipei Sun *, ${ }^{\dagger}$ \\ tDepartment of Electronics and Nanoengineering, Aalto University, Espoo, FI-00076, Finland \\ ‡Department of Applied Physics, Aalto University, Espoo, FI-00076, Finland \\ "Hefei National Laboratory for Physical Science at Microscale and Department of Physics, University of Science and \\ Technology of China, Hefei 230026, China \\ ${ }^{\perp}$ Key Laboratory of Strongly-Coupled Quantum Matter Physics, University of Science and Technology of China, Chi- \\ nese Academy of Sciences, Hefei 230026, China \\ §Collaborative Innovation Center of Advanced Microstructures, Nanjing 210093, China \\ ॠCambridge Graphene Centre, Department of Engineering, University of Cambridge, Cambridge CB3 0FA, UK
}

\begin{abstract}
Birefringence is an inherent optical property of anisotropic materials introduced by the anisotropic confinement in their crystal structures. It enables manipulation of light propagation properties (e.g., phase velocity, reflection, and refraction) for various photonic and optoelectronic applications, including waveplates and liquid crystal displays. Two-dimensional (2D) layered materials with high anisotropy are currently gaining an increasing interest for polarization-integrated nanodevice applications, which advances the research on birefringent materials. In this article, we investigate the optical birefringence of three anisotropic 2D layered materials (black phosphorus (BP), rhenium disulfide $\left(\mathrm{ReS}_{2}\right)$, and rhenium diselenide $\left.\left(\operatorname{ReSe}_{2}\right)\right)$. We demonstrate that the birefringence in BP $(\sim 0.245)$ is $\sim 6$ times larger than that of $\operatorname{ReS}_{2}(\sim 0.037)$ and $\operatorname{ReSe}_{2}$ $(\sim 0.047)$ at $520 \mathrm{~nm}$ and is comparable to the current state of the art bulk materials (e.g., $\left.\mathrm{CaCO}_{3}\right)$. We use these $2 \mathrm{D}$ materials to fabricate atomically-thin optical waveplates and investigate their performance. In particular, for BP, we observe a polarization-plane rotation of $\sim 0.05^{\circ}$ per atomic layer at $520 \mathrm{~nm}$. Our results show that the relatively large birefringence of anisotropic 2D layered materials can enable accurate manipulation of light polarization with atomically controlled device thickness for various applications where integrated, nanoscale polarization-controllers are required.
\end{abstract}

KEY WORDS: anisotropic two-dimensional layered materials, black phosphorus, rhenium disulfide, rhenium diselenide, anisotropic, birefringence, waveplates

Recently, anisotropic two-dimensional (2D) layered materials are gaining an increasing amount of interest to fabricate anisotropic nano-devices for various applications. For example, black phosphorus (BP),1-6 the most widely studied anisotropic 2D material, has a puckered hexagonal crystal structure with two non-equivalent in-plane crystal directions (armchair (AC) and zigzag (ZZ) ${ }^{7}$; Figure 1a), different from graphene ${ }^{8-10}$ and typical transitional metal dichalcogenides (e.g., $\mathrm{MoS}_{2}$ and $\mathrm{WSe}_{2}$ ). ${ }^{11-20}$ As with the crystal structure, the physical properties of BP (e.g., electrical, ${ }^{2}$ mechanical, ${ }^{4}$ thermal, ${ }^{21}$ ) are also anisotropic. $\mathrm{ReS}_{2}$ and $\mathrm{ReSe}_{2}$ are two examples of other anisotropic 2D material. They also demonstrate anisotropic responses arising from their inplane anisotropic structure.22-28 These 2D materials also have been demonstrated to have a strong anisotropic optical conductivity. For example, BP exhibits anisotropic linear (e.g., absorption, ${ }^{29}$ photoluminescence, ${ }^{7,30,31}$ optical contrast, ${ }^{29}$ and optical phase ${ }^{32}$ ) and nonlinear optical responses (e.g., Raman scattering,,33-38 saturable absorption, ${ }^{39}$ and harmonic generation ${ }^{40}$ ). These anisotropic responses open up possibilities for integrated, polarization-dependent, novel optical devices, including polarization-sensitive broadband photodetectors, ${ }^{31,} 41$ and linearly-polarized ultrafast lasers. ${ }^{39,} 42$ However, such devices have not yet been realised. In this article, we demonstrate atomically-thin optical waveplates based on the optical birefringence of three typical anisotropic 2D materials (BP, $\mathrm{ReS}_{2}$ and $\mathrm{ReSe}_{2}$ ) in the visible spectral range. Among the three materials investigated, the waveplate performance (i.e., phase retardance, polarization-plane rotation angle) from $\mathrm{BP}$ is superior to that of $\operatorname{ReS}_{2}$ and $\mathrm{ReSe}_{2}$. Our experiments show that the birefringence in BP $(\sim 0.245)$ is $\sim 6$ times larger than that in $\mathrm{ReS}_{2}(\sim 0.037)$ and $\operatorname{ReSe}_{2}(\sim 0.047)$ at $520 \mathrm{~nm}$ wavelength. We also demonstrate a polarization-plane rotation of $\sim 0.05^{\circ}, 0.02^{\circ}$ and $0.011^{\circ}$ per atomic layer (AL) at the same wavelength when linearly polarized light propagates through $\mathrm{BP}, \mathrm{ReS}_{2}$ and $\mathrm{ReSe}_{2}$ flakes, respectively. Our results show that the performance on polarization control from BP is comparable to that of the current state of the art bulk materials (e.g. $\mathrm{CaCO}_{3}$ ) and could be very attractive for integrated polarization-controllers (e.g. waveplates, phase 
compensator), where the light polarization state requires precise adjustments at the nanometer scale.

\section{RESULTS AND DISCUSSION}

Crystal orientation determination. All the samples studied in this work are fabricated using the micromechanical exfoliation method ${ }^{43,44}$ and then transferred onto transparent quartz substrates. The thickness of the flakes is measured by Atomic Force Microscopy, with detailed measured results shown in Supporting Information (SI).

Like other anisotropic 2D materials, the highly asymmetric crystal structure in $\mathrm{BP}$ enables its strong in-plane anisotropic optical response. For example, the Raman spectra of BP show strong polarization dependence, similar to one-dimensional nanomaterials (e.g. carbon nanotubes ${ }^{45-47}$ ). This can be used to determine the crystal orientation of BP and other anisotropic crystals. ${ }^{33-35}$ Typical Raman spectrum (Figure S1 in SI) of a BP flake shows three Raman modes at $363 \mathrm{~cm}^{-1}, 440 \mathrm{~cm}^{-1}$, and $468 \mathrm{~cm}^{-1}$, corresponding to the $A_{g}^{1}$, $B_{2 g}$, and $A_{g}^{2}$ vibration modes, respectively. ${ }^{48}$ The atomic motion associated with the $A_{g}^{2}$ mode occurs primarily along the AC direction. Thus the associated Raman scattering intensity is the strongest when the excitation laser polarization is aligned with the AC direction. ${ }^{33}$ The intensity of the $A_{g}^{2}$ mode can therefore be used to determine the crystal orientations of the BP flakes. In our polarization-resolved Raman experiment, the excitation light is linearly polarized and the polarization analyser is kept parallel to the polarization direction of the excitation light. The crystal orientations are then determined by rotating the BP flakes and measuring the angular dependence of Raman scattering intensity of the in-plane $A_{g}^{2}$ mode. As measured, the angular dependence of the $A_{g}^{2}$ mode intensity of the selected BP flake is shown in Figure 1b. This demonstrates that the AC direction of this BP sample is parallel with the direction of the excitation laser polarization when the BP flake is rotated by $90^{\circ}$ (or $270^{\circ}$ ). Using a similar strategy, we also use Raman to determine the crystal orientation of $\mathrm{ReS}_{2}$ and $\mathrm{ReSe}_{2}$. Details on the polarization-depended Raman measurements of the three crystals are presented in SI.

Polarization-resolved optical microscopy (PROM) is an alternative tool to determine the crystal orientation of anisotropic crystals. ${ }^{34}$ Figure 1c shows the transmitted PROM images of a BP flake as a function of the flake rotation angle under crossed-polarized light illumination. In this case, the polarizer and the analyser in the PROM are set perpendicular to each other. The flake image is recorded by a CCD camera with $15^{\circ}$ rotation steps. The flake brightness appears the highest when the crystal axis direction is $45^{\circ}$ (and $135^{\circ}$ ) with respect to the incident polarization direction, while no contrast from the BP flake is observed when one of the crystal axes is parallel to the polarizer direction (i.e., $0^{\circ}$ and $90^{\circ}$ ). This phenomenon originates from the birefringence of BP crystals. It could be explained as follows: when the polarized direction of the incident light is along the AC (or ZZ) direction, the output polarization is unchanged, still perpendicular to the polarization direction of (a)

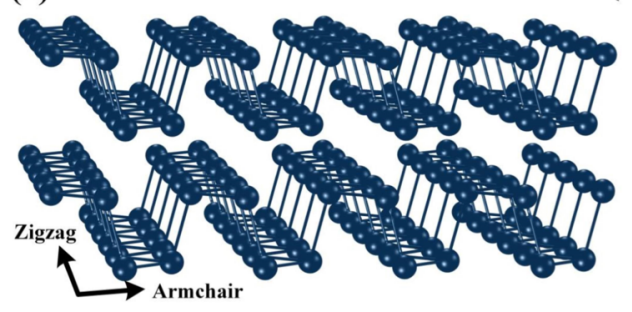

(b)

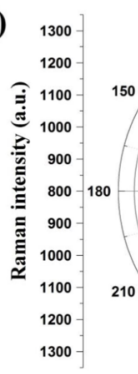

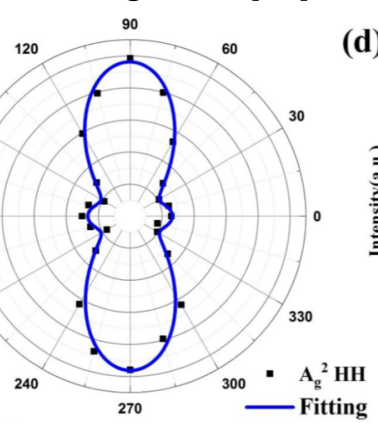
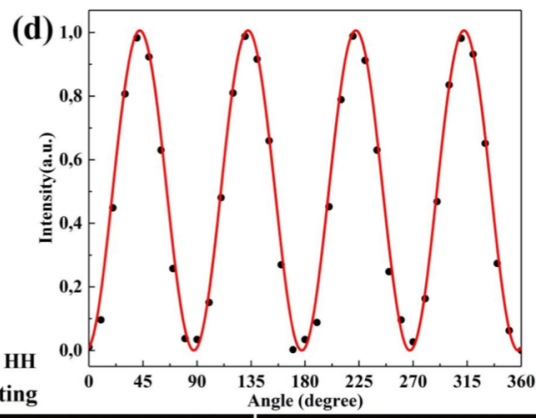
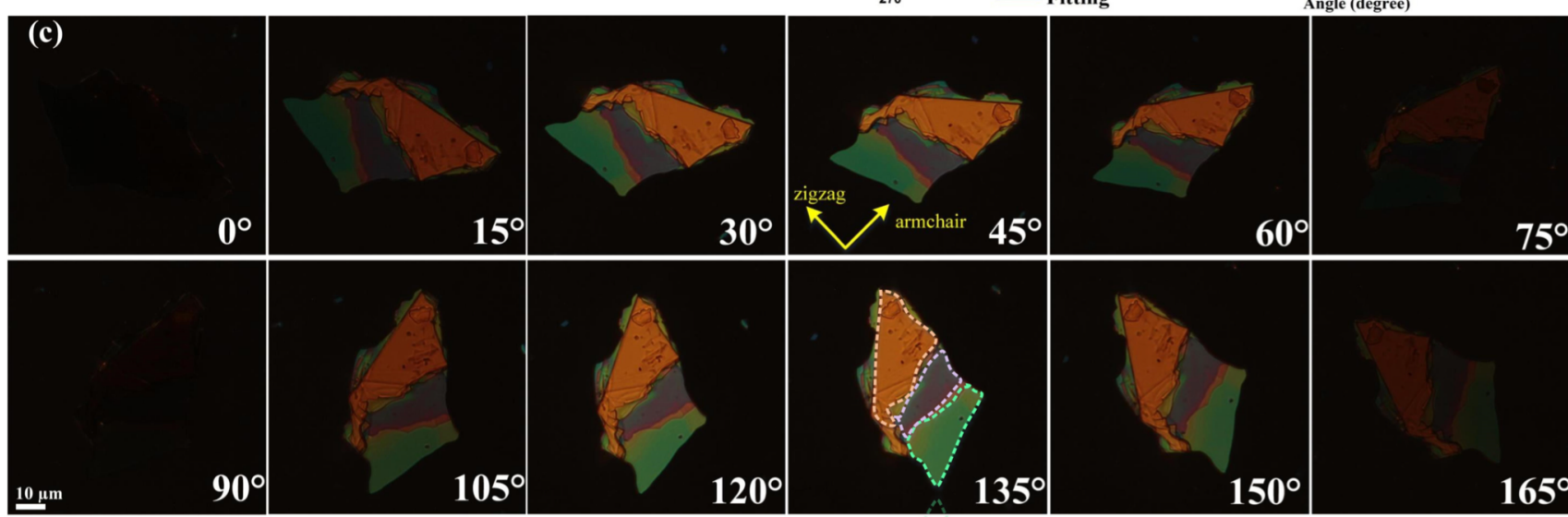

Figure 1 Anisotropic optical properties of BP. (a) Schematic structure of BP; (b) $A_{g}^{2}$ mode intensity with different input polarization angles. Both polarization directions of the excitation laser and the analyser are horizontal in the polarized Raman measurement. (c) Transmitted polarization-resolved optical images under crossed-polarized light illumination in the PROM (i.e., the polarization direction of the incident light and the polarization analyser is perpendicular to each other) with the BP flake rotation angle from $0^{\circ}$ to $180^{\circ}$. The step of rotation angle is $15^{\circ}$. The thickness of the flake area marked with the green, purple and yellow boxes is $\sim 45,35$ and $220 \mathrm{~nm}$, respectively. (d) The transmitted light intensity as a function of BP rotation angle (corresponding to Figure 1c) under crossed polarization illumination. The red line depicts the fitting result. 


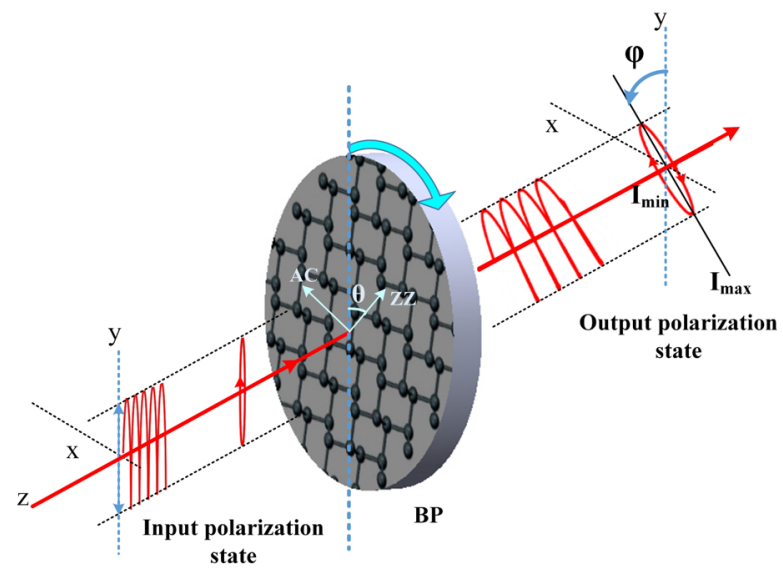

Figure 2 Schematic diagram of the birefringence measurement. A nearly linearly polarized input light has an incident angle $\theta$ (with measurement error of $\pm 1^{\circ}$ ) with respect to the BP's ZZ axis. After propagating through the BP flake, the incident polarization state typically changes to be elliptical, and the output polarization-plane rotates by an angle of $\varphi$ (with measurement error of $\pm 1^{\circ}$ ). $I_{\max }$ and $I_{\min }$ correspond to the maximum and minimum transmitted intensity that is experimentally measured by rotating a polarization analyzer.

the analyser. Therefore, the image is dark. However, when the incident polarization direction has an angle of $45^{\circ}$ (or $135^{\circ}$ ) to the BP crystal axis, the light intensity experiences the maximum phase retardance. This changes the incident polarization state into the elliptical. Thus the light intensity along the polarization direction of the analyser increases to the maximum, corresponding to the brightest flake image. Similarly, for the case of reflection mode, the brightness of the images also changes with the rotation angle of the flake (see Figure S3 in SI). Note that the flake colors at different flake thicknesses are different under the same measurement conditions. For example, Figure 1c mainly presents three colors - green, yellow and purple (marked with different dotted boxes), originating from the interference effect on the flake with different thicknesses. ${ }^{49}$ Using these colors, it is possible to estimate the sample thickness. ${ }^{50,51}$

For $\mathrm{ReS}_{2}$ and $\mathrm{ReSe}_{2}$, similar measurements on their PROM images (Figure $\mathrm{S} 5$ for $\mathrm{ReS}_{2}$, Figure $\mathrm{S} 7$ for $\mathrm{ReSe}_{2}$ ) are also carried out (details in SI). The optical contrast from $\mathrm{ReS}_{2}$ and $\mathrm{ReSe}_{2}$ is weaker than that from BP when the PROM images are compared. This suggests that the birefringence of $\mathrm{ReS}_{2}$ and $\mathrm{ReSe}_{2}$ is smaller than that of BP.

To quantitatively describe the birefringence-induced transmitted light intensity change, we use a custom-build PROM to measure the maximum transmitted light intensity as a function of the flake rotation angle (see Figure $1 \mathrm{~d}$ ). The diameter of the beam spot on the flakes is $\sim 10 \mu \mathrm{m}$, much smaller than the 2D flakes we use in the experiments. As shown in Figure 1d, the transmitted intensity changes periodically with the flake rotation angle with a period of $90^{\circ}$. Further, the transmitted intensity is the lowest when the BP flake is rotated by 0 and $90^{\circ}$. When the flake is rotated by $45^{\circ}$ with respect to the incident light polarization, the transmitted light intensity through the BP flake is the highest, as illustrated in Figure 1c. These results suggest strong bire- fringence of the BP flakes. This is consistent with the discussion above and can be used for the determination of the crystal axis orientation. The red line in Figure $1 \mathrm{~d}$ is the fitting of the transmitted light intensity following the equation $T=\alpha \sin ^{2}(2 \theta)$, where the coefficient $\alpha$ is the total transmittance along the AC and ZZ axes. The fit gives $\alpha=$ $\sim 0.97$ for our $\sim 45 \mathrm{~nm}$ BP flake, consistent with previous absorption measurement results. ${ }^{32}$ We also measure other anisotropic (i.e. $\mathrm{ReS}_{2}$ and $\mathrm{ReSe}_{2}$ ) and isotropic (e.g., $\mathrm{MoS}_{2}$ ) 2D materials. Similar intensity modulation property (as shown in Figure 1d) is also observed with $\mathrm{ReS}_{2}$ and $\mathrm{ReSe}_{2}$, but not with $\mathrm{MoS}_{2}$. This highlights the intrinsic optical birefringence of the anisotropic 2D materials we study here. Nevertheless, we note that the crystal orientation of $\mathrm{BP}, \mathrm{ReS}_{2}$ and $\mathrm{ReSe}_{2}$ can be determined by the PROM approach. The results from the PROM method also match well with the Raman measurements (Table S1 in SI). However, compared with the Raman method, the PROM approach is more cost-effective as it doesn't need a specific laser source and a spectrum analyser which are typically needed in Raman spectroscopy.

Birefringence of anisotropic 2D materials. To quantitatively characterize the birefringence of the anisotropic 2D materials we study, we measure the phase shift (i.e., the phase retardance) between two polarization components projected along two different crystallographic orientations when a linearly-polarized beam is passed through the materials; see the schematic diagram in Figure 2. The polarization of the transmitted light is examined through a polarization analyser. By rotating the analyser (Figure S8 in SI), the maximum $\left(I_{\max }\right)$ and minimum $\left(I_{\min }\right)$ transmitted light power is recorded by a power detector to analyze the polarization state of the output light. In this case, $I_{\max }$ stands for the intensity along the long-axis (i.e., ZZ direction), while $I_{\min }$ for the short-axis (i.e., AC direction). Therefore, the polarization ellipticity $(e)$ of the light after passing through the flake can be calculated by the intensity ratio between the long and short axes (i.e., $e=I_{\max } / I_{\min }$ ) for birefringence characterization.

Figure 3a presents the ellipticity change as a function of the flake rotation angle $(\theta)$ after the light passes through a $\sim 45$ $\mathrm{nm}$ thick BP flake at three different wavelengths. It can be seen that the ellipticity is the highest when the flake rotation angle $(\theta)$ is either $0^{\circ}$ or $90^{\circ}$. This is expected as no phase retardance occurs. In this case, when the incident light is linearly polarized along the ZZ (or AC) direction, no light is projected along the AC (or ZZ) direction. Thus $I_{\min }$ is nearly zero when rotating the analyser. However, the ellipticity values at $\theta=0^{\circ}$ and $90^{\circ}$ are different. For instance, the ellipticity is $\sim 1522$ at $\theta=0^{\circ}$, and $\sim 850$ at $\theta=90^{\circ}$ at $520 \mathrm{~nm}$ wavelength (Figure $3 \mathrm{a}$ ). This is because of the dichroic absorption (the absorption difference along two crystal axes) of BP. Comparing the ellipticity values at these two angles (i.e., $\theta=0^{\circ}$ and $90^{\circ}$ ), the magnitude of the dichroic absorption can be extracted. From this, we find that the transmittance ratios between $\mathrm{AC}$ and $\mathrm{ZZ}$ axes are $\sim 88.7 \%, \sim 93.0 \%$, and $\sim 95.9 \%$ for $520 \mathrm{~nm}, 642 \mathrm{~nm}$, and $730 \mathrm{~nm}$, respectively. This agrees well with the previous measurement. ${ }^{29}$ On the other hand, when $\theta$ is in between $0^{\circ}$ and $90^{\circ}$, the ellipticity value decreases rapidly due to the birefringence. Note that we also measure the ellipticity change of two isotropic $\mathrm{MoS}_{2}$ 

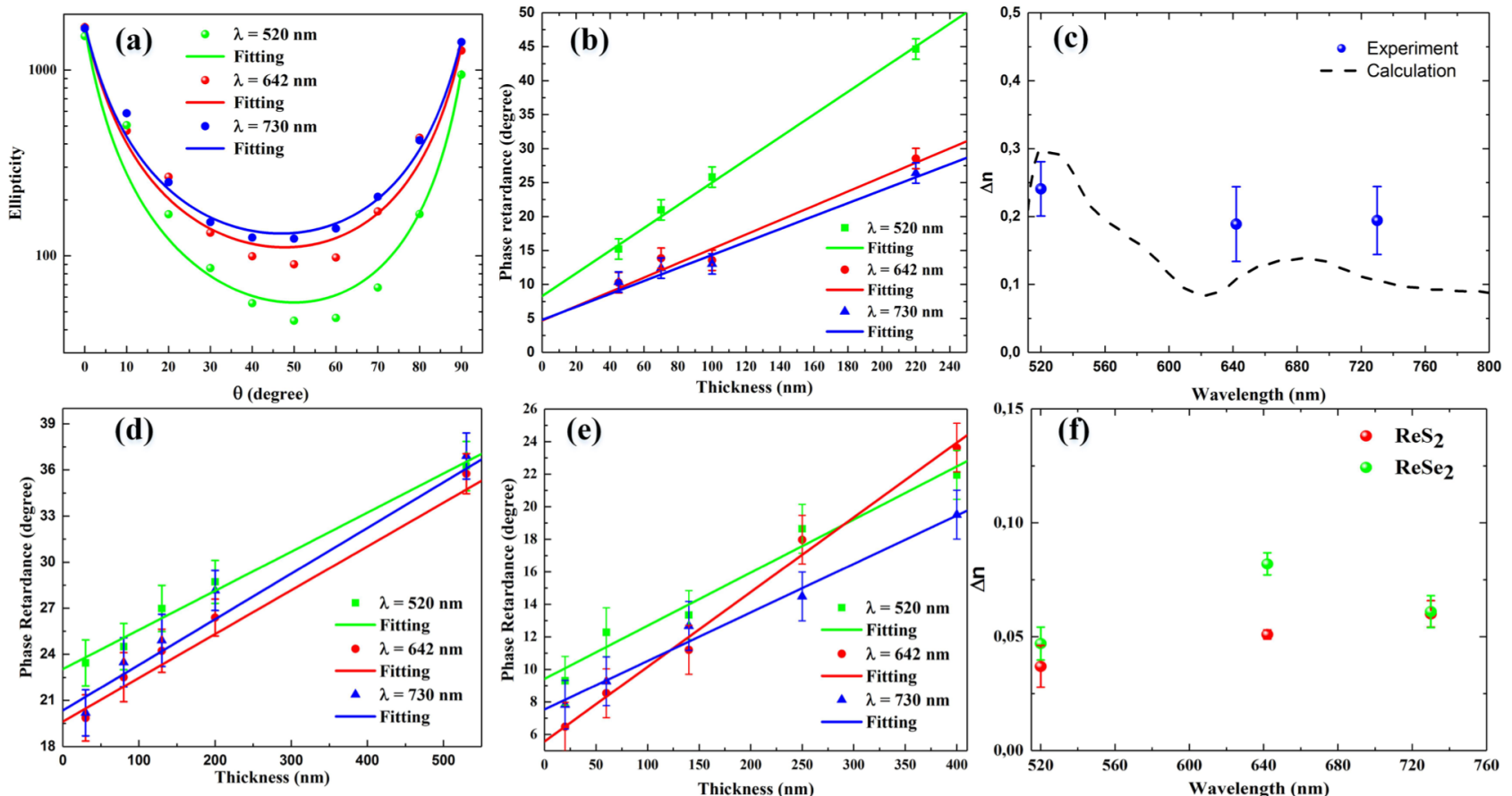

Figure 3 Birefringence of anisotropic 2D materials. (a) The measured light ellipticity (dots) and its fitting (lines, following eq 1) as a function of rotation angle after the light propagates through a $45 \mathrm{~nm}$ BP flake at different wavelengths. (b) Phase retardance as a function of BP flake thickness. (c) Birefringence $(\Delta \mathrm{n})$ of BP at different wavelengths (Experimental results: Dots; Theoretical results: lines). The phase retardance and their fittings as a function of $\operatorname{ReS}_{2}$ (d) and $\operatorname{ReSe}_{2}(\mathrm{e})$ flake thickness at three wavelengths in the visible range. (f) Experimental results of the birefringence of $\mathrm{ReS}_{2}$ and $\mathrm{ReSe}_{2}$.

flakes (Figure S9 in SI), showing that the ellipticity value remains constant with the rotation of the flake. This confirms that only anisotropic 2D materials have birefringence.

To accurately obtain the value of the birefringence, we use $\mathrm{BP}$ as an example to fit the ellipticity change (Figure 3a), using the following equation:

$$
\sqrt{\mathrm{e}}=\frac{1+s \sqrt{1-4 \sin ^{2} \delta \frac{A_{Z Z}^{2} A_{A C}^{2}}{\left(A_{Z Z}^{2}+A_{A C}^{2}\right)^{2}}}}{1-s \sqrt{1-4 \sin ^{2} \delta \frac{A_{Z Z}^{2} A_{A C}^{2}}{\left(A_{Z Z}^{2}+A_{A C}^{2}\right)^{2}}}}
$$

where $\mathrm{s}=1$ for $A_{Z Z}^{2}-A_{A C}^{2} \geq 0$ and $\mathrm{s}=-1$ for $A_{Z Z}^{2}-A_{A C}^{2}<0 . A_{A C}$ and $A_{z Z}$ are the amplitude of the transmitted light projected along the $\mathrm{AC}$ and $\mathrm{ZZ}$ directions, respectively, with the consideration of $\theta$ and the transmission coefficient along the $\mathrm{AC}$ and $\mathrm{ZZ}$ crystal axes separately. $\delta$ is the phase retardance between the $\mathrm{AC}$ and $\mathrm{ZZ}$ axes after light propagates through BP flakes. In our model, the incident light is normal to the flake surface, and the dichroic absorption is also considered in the fit. Detailed discussion on the model and eq 1 is presented in SI.

The ellipticity fitting of the $\sim 45 \mathrm{~nm}$ thick BP flake is presented in Figure 3a by solid lines, matching well with the measured ellipticity values. The fitted phase retardance $\delta$ with eq 1 at three wavelengths is plotted in Figure 3b, where the error bars are from the deviation of the fitting. In addition, BP flakes with other different thicknesses (i.e., 70 $\mathrm{nm}, 100 \mathrm{~nm}$, and $220 \mathrm{~nm}$ ) are also characterized and shown for comparison (see Figure S10 in SI). As seen in Figure 3b, the phase retardance increases linearly with the flake thickness, following the equation:

$$
\delta(d)=\frac{2 \pi \times \Delta n}{\lambda} d+\delta_{0}
$$

Table 1. Comparison of $\Delta \mathrm{n}$ from different materials

$\begin{array}{ccccc} & 520 \mathrm{~nm} & 642 \mathrm{~nm} & 730 \mathrm{~nm} & \text { Reference } \\ \mathrm{BP} & 0.245 \pm 0.04 & 0.196 \pm 0.055 & 0.202 \pm 0.05 & \text { This work } \\ \mathrm{ReS}_{2} & 0.037 \pm 0.009 & 0.051 \pm 0.002 & 0.060 \pm 0.006 & \text { This work } \\ \mathrm{ReSe}_{2} & 0.047 \pm 0.007 & 0.082 \pm 0.004 & 0.061 \pm 0.007 & \text { This work } \\ \mathrm{BP} & 0.23 & 0.12 & & \text { Ref. 29 } \\ \mathrm{CaCO}_{3} & & 0.172(590 \mathrm{~nm}) & & \text { Ref. 56 } \\ \mathrm{LiNbO}_{3} & & 0.085(590 \mathrm{~nm}) & & \text { Ref. 56 } \\ \mathrm{SiO}_{2} & & 0.009(590 \mathrm{~nm}) & & \text { Ref. 56 }\end{array}$

where $\delta(d)$ is the fitted phase retardance, $\delta_{0}$ is the phase retardance of the incident polarized light before the BP flake, $\Delta \mathrm{n}$ is the birefringence, characterized by the refractive index difference between two perpendicular crystal axes, $d$ is the flake thickness and $\lambda$ is the wavelength of the incident light. As such, the slope (i.e., $(2 \pi \times \Delta n) / \lambda)$ reveals the magnitude of the birefringence. We note that this equation assumes that the material birefringence is independent of the material thickness. This is a reasonable assumption as the thicknesses of our studied flakes are $>40 \mathrm{~nm} .^{52}$ By subtracting $\delta_{0}$ (i.e., $d=0$ ), the phase retardance resulting from BP's birefringence is extracted in Figure S11 (see SI). A maximum retardance of $38^{\circ}$ is obtained at $520 \mathrm{~nm}$ wavelength with a $220 \mathrm{~nm}$ thick BP flake. Given that the monolayer thickness of $\mathrm{BP}$ is $0.53 \mathrm{~nm},{ }^{30}$ we estimate a phase retardance of $\sim 0.1^{\circ}$ (corresponding to $\sim \lambda / 3600$ ) per atomic layer (AL). The refractive index difference between the $\mathrm{AC}$ and ZZ crystal directions (i.e., $\Delta \mathrm{n}$, the birefringence) in BP is calculated from the experimental phase retardance results with eq 2 and presented in Figure $3 \mathrm{c}$ as a function of the wavelength. We find that $\Delta \mathrm{n}$ is similar $(\sim 0.20)$ at 642 and $730 \mathrm{~nm}$ wavelengths, but is larger $(\sim 0.24)$ at $520 \mathrm{~nm}$. This is comparable to the previously reported results on $\mathrm{BP}^{29}$ (a detailed com 

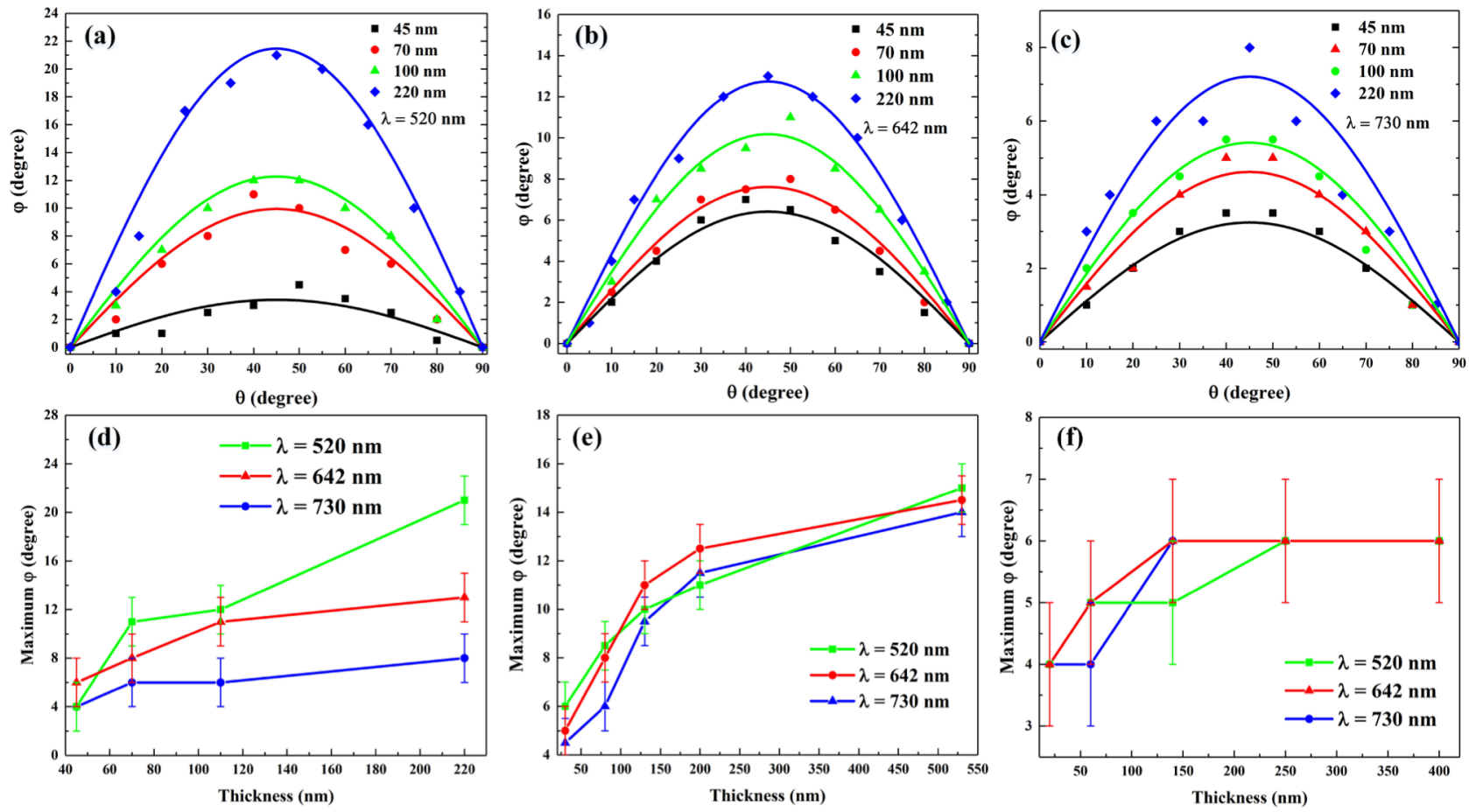

Figure 4 Anisotropic 2D materials based waveplates. Dependence of the polarization-plane rotation angles $(\varphi)$ for different BP thickness flakes at (a) $520 \mathrm{~nm}$ (b) $642 \mathrm{~nm}$ and (c) $730 \mathrm{~nm}$ separately. The dots are experimental data, while the curves are plotted here to guide the eye. (d), (e) and (f) illustrate the maximum polarization-plane rotation angles ( $\varphi$ ) as a function of $B P, \operatorname{ReS} 2$ and $\mathrm{ReSe}_{2}$ flake thickness in the visible range, respectively.

parison in Table 1). We also present the theoretically calculated $\Delta \mathrm{n}$ of BP from the dielectric functions within the independent particle approximation on top of the electronic structure that is obtained using hybrid density functions (details in SI). ${ }^{53}$ As shown in Figure 3c, the experimentally measured and the theoretically calculated birefringence are in good agreement.

The same birefringence measurement method is also employed for $\mathrm{ReS}_{2}$ and $\mathrm{ReSe}_{2}$. The experimental results and the ellipticity fits are shown in Figures S13 (for ReS2) and S14 (for $\mathrm{ReSe}_{2}$ ) in SI. The fitted phase retardance as a function of the flake thickness at three wavelengths is presented in Figure 3d (for $\mathrm{ReS}_{2}$ ) and Figure 3e (for $\mathrm{ReSe}_{2}$ ). As expected, the two figures illustrate that the phase retardance increases linearly with the flake thickness. By subtracting $\delta_{0}$ from the incident light with eq 2 , we obtain the birefringence induced phase retardance (Figure $\mathrm{S} 13$ for $\mathrm{ReS}_{2}$ and $\mathrm{S} 14$ for $\mathrm{ReSe}_{2}$ in $\mathrm{SI}$ ). The results give the maximum retardance of $\sim 0.021^{\circ} / \mathrm{AL}$ (at the wavelength of $730 \mathrm{~nm}$ ) for $\mathrm{ReS}_{2}$ and $\sim 0.032^{\circ} / \mathrm{AL}$ for $\operatorname{ReSe}_{2}$ (at the wavelength of $642 \mathrm{~nm}$ ), respectively, considering that the atomic layer thickness of both $\mathrm{ReS}_{2}$ and $\mathrm{ReSe}_{2}$ is $\sim 0.7 \mathrm{~nm} .{ }^{54}$ The birefringence of $\mathrm{ReS}_{2}$ and $\mathrm{ReSe}_{2}$ is then calculated from the experimental phase retardance results with eq 2 , and plotted in Figure 3f. This shows that $\Delta \mathrm{n}$ is depended on the wavelength but the difference between $\mathrm{ReS}_{2}$ and $\mathrm{ReSe}_{2}$ is small $(<0.03)$. We propose that this is because the atomic structures and properties of $\mathrm{ReS}_{2}$ and $\mathrm{ReSe}_{2}$ are similar. ${ }^{55}$ Note that we are unable to use the density functional theory calculation method (details in SI) used for BP for the case of $\mathrm{ReS}_{2}$ and $\mathrm{ReSe}_{2}$. This is due to their large unit cell and distorted structures. Such theoretical birefringence calculation of $\mathrm{ReS}_{2}$ and $\mathrm{ReSe}_{2}$ requires further study.
For comparison, we list $\Delta \mathrm{n}$ of $\mathrm{BP}, \mathrm{ReS}_{2}$, and $\mathrm{ReSe}_{2}$, in Table 1. By comparing $\Delta \mathrm{n}$ of these three anisotropic $2 \mathrm{D}$ materials, we find that the birefringence of $\mathrm{BP}$ is $\sim 6$ times larger than that of $\mathrm{ReS}_{2}$ and $\mathrm{ReSe}_{2}$ at $520 \mathrm{~nm}$. This is consistent with our previous PROM results (Figure S3 for BP, Figure S5 for $\mathrm{ReS}_{2}$, Figure S7 for $\mathrm{ReSe}_{2}$ ), which reveal that the highest contrast (i.e., the largest birefringence) observed among the studied 2D materials in this work comes from BP. Birefringence values of three anisotropic bulk optical materials are also listed in Table 1 . This also shows that $\Delta \mathrm{n}$ of BP is comparable to that of the bulk commercial anisotropic materials (e.g., $\left.\mathrm{CaCO}_{3}\right) \cdot{ }^{56}$

Waveplates based on anisotropic 2D materials. Birefringence is widely used to make optical waveplates for polarization related applications (e.g., manipulating the polarization state of light ${ }^{57}$ ). Here, we utilize the birefringence of anisotropic 2D materials for waveplate applications. To measure the rotation of polarization-plane, a key parameter of optical waveplates, we characterize the polarization rotation angle $\varphi$ of the transmitted light induced by the birefringence (as shown in Figure 2).

Figures $4 \mathrm{a}-\mathrm{c}$ illustrate the polarization-plane rotation angle of the incident light as a function of the BP flake rotation angle $(\theta)$ at three different wavelengths. As illustrated, $\varphi$ increases with the flake thickness, as the optical path length is longer in the thicker flakes. To clarify the wavelength dependence of $\varphi$, the experimentally measured maximum $\varphi$ values at $520 \mathrm{~nm}, 642 \mathrm{~nm}$, and $730 \mathrm{~nm}$ are presented in Figure $5 \mathrm{~d}$. As observed from the flakes with different thicknesses, $\varphi$ at $520 \mathrm{~nm}$ wavelength is larger than that at 642 $\mathrm{nm}$, and is the smallest at $730 \mathrm{~nm}$. The maximum polarization rotation angle at $520 \mathrm{~nm}$ wavelength is $21^{\circ}$ for the 220 
$\mathrm{nm}$ thick flake, corresponding to a polarization rotation angle of $\sim 0.05^{\circ} / \mathrm{AL}$. In addition, $\Delta \mathrm{n}$ is wavelength dependent (Figure 3a), indicating chromatic dispersion of BP. This explains the wavelength dependence of $\varphi$ in Figure 4d. Our results show that the polarization parameters such as phase retardance and polarization-plane rotation can be adjusted with BP flakes, opening its potential for various applications, including optical waveplates as demonstrated here.

The rotation angle of the polarization-plane as a function of the flake thickness of $\mathrm{ReS}_{2}$ and $\mathrm{ReSe}_{2}$ at three wavelengths is presented in Figures 4e-f, respectively. Similar to what is observed in BP, $\varphi$ increases with the flake thickness. However, the small difference in $\varphi$ between $\operatorname{ReS}_{2}$ and $\operatorname{ReSe}_{2}$ indicates that the birefringence of $\mathrm{ReSe}_{2}$ is slightly larger than that of $\mathrm{ReS}_{2}$. This agrees well with the previous birefringence measurement (as listed in Table 1). By comparing Figure $4 \mathrm{~d}$ to Figures $4 \mathrm{e}-\mathrm{f}, \varphi$ of $\mathrm{ReS}_{2}$ and $\mathrm{ReSe}_{2}$ is much smaller than that from BP. This confirms that BP has a larger birefringence than $\mathrm{ReS}_{2}$ and $\mathrm{ReSe}_{2}$. Therefore, BP offers better polarization control performance over these two anisotropic 2D crystals. We note that there are many other anisotropic 2D layered materials (e.g., GeS, ${ }^{57} \mathrm{GaTe},{ }^{58} \mathrm{SnS}, 59$ and $\mathrm{SnSe}^{60}$ ), which have been studied to show anisotropic physical responses due to their anisotropic crystal structures. The intrinsic optical birefringence properties of these 2D layered materials also deserve further investigation.

\section{CONCLUSIONS}

We demonstrate atomically-thin optical waveplates based on the birefringence of three anisotropic 2D layered materials (BP, $\mathrm{ReS}_{2}$, and $\mathrm{ReSe}_{2}$ ). With $\mathrm{BP}$, we observe a polarization-plane rotation of up to $\sim 0.05^{\circ}$ per atomic layer at $520 \mathrm{~nm}$. We find that the birefringence of BP is comparable to that of commercially used bulk birefringent materials such as $\mathrm{CaCO}_{3}$ and is $\sim 6$ times larger than that of $\mathrm{ReS}_{2}$ and $\mathrm{ReSe}_{2}$. Our results highlight that the relatively large birefringence of anisotropic 2D layered materials such as BP can facilitate accurate manipulation of light polarization with atomically controlled device thickness for various applications where integrated polarization-controllers at the nanoscale are required.

\section{METHODS}

Sample preparation and characterization. All flakes (BP, $\mathrm{ReS}_{2}, \mathrm{ReSe}_{2}$ ) are produced from their bulk crystals by mechanical exfoliation and then transferred onto transparent quartz substrates. The transferred flakes are first characterized by polarized Raman microscopy (WITec Alpha 300 RA) to determine the crystal orientation. Then, a PROM (Leica DM4500) is used to analyse the crystal orientation and capture the optical images under reflection and transmission modes. The custom-built PROM is equipped with fiber coupled visible lasers (Thorlabs, MCLS1) for measurement. A 20X, NA=0.4 objective is used to focus the beam on the flake with $\sim 10 \mu \mathrm{m}$ diameter. During the experiments, all 2D flake samples are characterized immediately after preparation and then kept in the nitrogen box to avoid degradation and/or contamination.
Birefringence measurement process. Three monochromatic continuous wave (CW) lasers at $520 \mathrm{~nm}, 642 \mathrm{~nm}$, and 730 $\mathrm{nm}$ are used as the light sources to test the birefringence of the 2D materials. A polarizer is inserted between the laser source and the flakes to obtain an approximately linearly (with extinction ratio $>30 \mathrm{~dB}$ ) polarized beam incident on the sample. The exfoliated samples are placed on a rotation stage to change the sample orientation. $\theta$ describes the rotation angle between the polarization direction of the incident light and crystal orientation of the flake. After passing through the BP flake, the polarization of the light is examined through a polarization analyser. The schematic diagram of the detection process is illustrated in Figure S8 (see SI). By rotating the analyser, the maximum $\left(\boldsymbol{I}_{\max }\right)$ and minimum $\left(\boldsymbol{I}_{\min }\right)$ transmitted light power is recorded with a power detector to examine the ellipticity of the transmitted light. The angle measurement error is $\pm 1^{\circ}$.

\section{ASSOCIATED CONTENT}

\section{Supporting Information}

The Supporting Information is available free of charge on the ACS Publications website.

Polarization-dependent Raman spectra of $\mathrm{BP}, \mathrm{ReS}_{2}$ and $\mathrm{ReSe}_{2}$, Polarization-resolved optical images of the three materials, the fitting model and results for the phase change, theoretical calculation of the birefringence of black phosphorus (PDF).

\section{AUTHOR INFORMATION}

\author{
Corresponding Author \\ *E-mail: zhipei.sun@aalto.fi \\ ORCID \\ Zhipei Sun: 0000-0002-9771-5293 \\ Notes
}

The authors declare no competing financial interest.

\section{ACKNOWLEDGMENT}

The authors acknowledge funding from Academy of Finland (Grant Nos. 285972, 292600, 295777, 304666, 286279 and 251748), National Natural Science Foundation of China (Grant No. 11534010), the European Union's Seventh Framework Programme (Grant No. 631610), China Scholarship Council, the TEKES projects (OPEC), and the Nokia Foundation. TH acknowledges funding from RAEng through a research fellowship (Graphex). We also acknowledge the provision of technical facilities at Micronova, Nanofabrication Center of Aalto University, and CSC-IT Center for Science Ltd. for generous grants of computer time.

\section{REFERENCES}

(1) Xia, F.; Wang, H.; Jia, Y., Rediscovering black phosphorus as an anisotropic layered material for optoelectronics and electronics. Nat. Commun. 2014, 5, 4458.

(2) Liu, H.; Neal, A. T.; Zhu, Z.; Luo, Z.; Xu, X.; Tomanek, D.; Ye, P. D., Phosphorene: an unexplored 2D semiconductor with a high hole mobility. ACS Nano 2014, 8, 4033-4041.

(3) Li, L.; Kim, J.; Jin, C.; Ye, G. J.; Qiu, D. Y.; da Jornada, F. H.; Shi, Z.; Chen, L.; Zhang, Z.; Yang, F.; Watanabe, K.; Taniguchi, T.; Ren, W.; Louie, S. G.; Chen, X. H.; Zhang, Y.; Wang, F., Direct observation of the layer-dependent electronic structure in phosphorene. Nat. Nanotech. 2017, 12, 21-25. 
(4) Wei, Q.; Peng, X., the Superior mechanical flexibility of phosphorene and few-layer black phosphorus. Appl. Phys. Lett. 2014, 104, 251915.

(5) Wang, X.; Lan, S., Optical properties of black phosphorus. Adv. Opt. Photonics 2016, 8, 618.

(6) Tran, V.; Soklaski, R.; Liang, Y.; Yang, L., Layer-controlled band gap and anisotropic excitons in few-layer black phosphorus. Phys. Rev. B 2014, 89, 235319.

(7) Qiao, J.; Kong, X.; Hu, Z. X.; Yang, F.; Ji, W., High-mobility transport anisotropy and linear dichroism in few-layer black phosphorus. Nat. Commun. 2014, 5, 4475.

(8) Li, D.; Xue, H.; Qi, M.; Wang, Y.; Aksimsek, S.; Chekurov, N.; Kim, W.; Li, C.; Riikonen, J.; Ye, F.; Dai, Q.; Ren, Z.; Bai, J.; Hasan, T.; Lipsanen, H.; Sun, Z., Graphene actively Q-switched lasers. 2D Mater. 2017, 4, 025095.

(9) Ferrari, A. C. et.al., Science and technology roadmap for graphene, related two-dimensional crystals, and hybrid systems. Nanoscale 2015, 7, 4598-4810.

(10) Martinez, A.; Sun, Z., Nanotube and graphene saturable absorbers for fibre lasers. Nat. Commun., 2013, 7, 842-845.

(11) Mak, K. F.; Shan, J., Photonics and optoelectronics of 2D semiconductor transition metal dichalcogenides. Nat. Photon. 2016, 10, 216-226.

(12) Bonaccorso, F.; Sun, Z.; Hasan, T.; Ferrari, A. C., Graphene photonics and optoelectronics. Nat. Photon. 2010, 4, 611-622.

(13) Koppens, F. H.; Mueller, T.; Avouris, P.; Ferrari, A. C.; Vitiello, M. S.; Polini, M., Photodetectors based on graphene, other twodimensional materials and hybrid systems. Nat. Nanotech. 2014, 9, 780-793.

(14) Wang, Q. H.; Kalantar-Zadeh, K.; Kis, A.; Coleman, J. N.; Strano, M. S., Electronics and optoelectronics of two-dimensional transition metal dichalcogenides. Nat. Nanotech. 2012, 7, 699-712. (15) Xia, F.; Mueller, T.; Lin, Y. M.; Valdes-Garcia, A.; Avouris, P., Ultrafast graphene photodetector. Nat. Nanotech. 2009, 4, 839843.

(16) Yang, J.; Wang, Z.; Wang, F.; Xu, R.; Tao, J.; Zhang, S.; Qin, Q.; Luther-Davies, B.; Jagadish, C.; Yu, Z.; Lu, Y., Atomically thin optical lenses and gratings. Light Sci. Appl. 2016, 5, e16046.

(17) Sun, Z.; Martinez, A.; Wang, F., Optical modulators with 2D layered materials. Nat. Photon. 2016, 10, 227-238.

(18) Bonaccorso, F.; Sun, Z., Solution processing of graphene, topological insulators and other $2 \mathrm{~d}$ crystals for ultrafast photonics. Opt. Mater. Express 2014, 4, 63-78.

(19) A. Saynatjoki; L. Karvonen1; H. Rostami; A. Autere; S. Mehravar; A. Lombardo; R. A.Norwood; T. Hasan; N. Peyghambarian; H. Lipsanen; K. Kieu; A. C. Ferrari; M. Polini; Sun, Z., Ultra-strong nonlinear optical processes and trigonal warping in MoS2 layers. arXiv, 1608.04101.

(20) Karvonen, L.; Saynatjoki, A.; Huttunen, M. J.; Autere, A.; Amirsolaimani, B.; Li, S.; Norwood, R. A.; Peyghambarian, N.; Lipsanen, H.; Eda, G.; Kieu, K.; Sun, Z., Rapid visualization of grain boundaries in monolayer $\mathrm{MoS}_{2}$ by multiphoton microscopy. Nat. Commun. 2017, 8, 15714.

(21) Luo, Z.; Maassen, J.; Deng, Y.; Du, Y.; Garrelts, R. P.; Lundstrom, M. S.; Ye, P. D.; Xu, X., Anisotropic in-plane thermal conductivity observed in few-layer black phosphorus. Nat. Commun. 2015, 6, 8572.

(22) Aslan, O. B.; Chenet, D. A.; van der Zande, A. M.; Hone, J. C.; Heinz, T. F., Linearly Polarized Excitons in Single- and Few-Layer $\mathrm{ReS}_{2}$ Crystals. ACS Photonics 2016, 3, 96-101.

(23) Sim, S.; Lee, D.; Noh, M.; Cha, S.; Soh, C. H.; Sung, J. H.; Jo, M. H.; Choi, H., Selectively tunable optical Stark effect of anisotropic excitons in atomically thin $\mathrm{ReS}_{2}$. Nat. Commun. 2016, 7, 13569.

(24) Zhao, H.; Wu, J.; Zhong, H.; Guo, Q.; Wang, X.; Xia, F.; Yang, L.; Tan, P.; Wang, H., Interlayer interactions in anisotropic atomically thin rhenium diselenide. Nano Res. 2015, 8, 3651-3661.

(25) Cui, Q.; He, J.; Bellus, M. Z.; Mirzokarimov, M.; Hofmann, T.; Chiu, H. Y.; Antonik, M.; He, D.; Wang, Y.; Zhao, H., Transient Absorption Measurements on Anisotropic Monolayer ReS. Small 2015, 11, 5565-5571.
(26) Chenet, D. A.; Aslan, O. B.; Huang, P. Y.; Fan, C.; van der Zande, A. M.; Heinz, T. F.; Hone, J. C., In-Plane Anisotropy in Mono- and Few-Layer $\mathrm{ReS}_{2}$ Probed by Raman Spectroscopy and Scanning Transmission Electron Microscopy. Nano Lett. 2015, 15, 56675672.

(27) Lin, Y.-C.; Komsa, H.-P.; Yeh, C.-H.; Bjorkman, T.; Liang, Z.-Y.; Ho, C.-H.; Huang, Y.-S.; Chiu, P.-W.; Krasheninnikov, A. V.; Suenaga, K., Single-Layer $\mathrm{ReS}_{2}$ : Two-Dimensional Semiconductor with Tunable In-Plane Anisotropy. ACS Nano 2015, 9, 11249-11257.

(28) Cui, Q.; Muniz, R. A.; Sipe, J. E.; Zhao, H., Strong and anisotropic third-harmonic generation in monolayer and multilayer $\mathrm{ReS}_{2}$. Phys. Rev. B 2017, 95, 165406.

(29) Mao, N.; Tang, J.; Xie, L.; Wu, J.; Han, B.; Lin, J.; Deng, S.; Ji, W.; $\mathrm{Xu}, \mathrm{H} . ;$ Liu, K.; Tong, L.; Zhang, J., Optical Anisotropy of Black Phosphorus in the Visible Regime. J. Am. Chem. Soc. 2016, 138, 300-305.

(30) Wang, X.; Jones, A. M.; Seyler, K. L.; Tran, V.; Jia, Y.; Zhao, H.; Wang, H.; Yang, L.; Xu, X.; Xia, F., Highly anisotropic and robust excitons in monolayer black phosphorus. Nat. Nanotech. 2015, 10, 517-521.

(31) Yuan, H.; Liu, X.; Afshinmanesh, F.; Li, W.; Xu, G.; Sun, J.; Lian, B.; Curto, A. G.; Ye, G.; Hikita, Y.; Shen, Z.; Zhang, S. C.; Chen, X.; Brongersma, M.; Hwang, H. Y.; Cui, Y., Polarization-sensitive broadband photodetector using a black phosphorus vertical p-n junction. Nat. Nanotech. 2015, 10, 707-713.

(32) Lan, S.; Rodrigues, S.; Kang, L.; Cai, W., Visualizing Optical Phase Anisotropy in Black Phosphorus. ACS Photonics 2016, 3, 1176-1181.

(33) Ribeiro, H. B.; Pimenta, M. A.; Matos, C. J. S. d.; Moreira, R. L.; Rodin, A. S.; Zapata, J. D.; Souza, E. z. A. T. d.; Neto, A. H. C., Unusual angular dependence of the Raman response in black phosphorus. ACS Nano 2015, 9, 4270-4276.

(34) Kim, J.; Lee, J. U.; Lee, J.; Park, H. J.; Lee, Z.; Lee, C.; Cheong, H., Anomalous polarization dependence of Raman scattering and crystallographic orientation of black phosphorus. Nanoscale 2015, 7, 18708-18715.

(35) Wu, J.; Mao, N.; Xie, L.; Xu, H.; Zhang, J., Identifying the crystalline orientation of black phosphorus using angle-resolved polarized Raman spectroscopy. Angew. Chem. Int. Ed. Engl. 2015, 54, 2366-2369.

(36) Ling, X.; Huang, S.; Hasdeo, E. H.; Liang, L.; Parkin, W. M.; Tatsumi, Y.; Nugraha, A. R.; Puretzky, A. A.; Das, P. M.; Sumpter, B. G.; Geohegan, D. B.; Kong, J.; Saito, R.; Drndic, M.; Meunier, V.; Dresselhaus, M. S., Anisotropic Electron-Photon and ElectronPhonon Interactions in Black Phosphorus. Nano Lett. 2016, 16, 2260-2267.

(37) Mao, N.; Wu, J.; Han, B.; Lin, J.; Tong, L.; Zhang, J., BirefringenceDirected Raman Selection Rules in 2D Black Phosphorus Crystals. Small 2016, 12, 2627-2633.

(38) Zhang, S.; Yang, J.; Xu, R.; Wang, F.; Li, W.; Ghufran, M.; Zhang, Y. W.; Yu, Z.; Zhang, G.; Qin, Q.; Lu, Y., Extraordinary photoluminescence and strong temperature/angle-dependent Raman responses in few-layer phosphorene. ACS Nano 2014, 8, 9590-9596.

(39) Li, D.; Jussila, H.; Karvonen, L.; Ye, G.; Lipsanen, H.; Chen, X.; Sun, Z., Polarization and Thickness Dependent Absorption Properties of Black Phosphorus: New Saturable Absorber for Ultrafast Pulse Generation. Sci. Rep. 2015, 5, 15899.

(40) Mas-Balleste, R.; Gomez-Navarro, C.; Gomez-Herrero, J.; Zamora, F., 2D materials: to graphene and beyond. Nanoscale 2011, 3, 20-30.

(41) Guo, Q.; Pospischil, A.; Bhuiyan, M.; Jiang, H.; Tian, H.; Farmer, D.; Deng, B.; Li, C.; Han, S. J.; Wang, H.; Xia, Q.; Ma, T. P.; Mueller, T.; Xia, F., Black Phosphorus Mid-Infrared Photodetectors with High Gain. Nano Lett. 2016, 16, 4648-55.

(42) Li, D.; Del Rio Castillo, A. E.; Jussila, H.; Ye, G.; Ren, Z.; Bai, J.; Chen, X.; Lipsanen, H.; Sun, Z.; Bonaccorso, F., Black phosphorus polycarbonate polymer composite for pulsed fibre lasers. Appl. Mater. Today 2016, 4, 17-23. 
(43) Bonaccorso, F.; Lombardo, A.; Hasan, T.; Sun, Z.; Colombo, L.; Ferrari, A. C., Production and processing of graphene and $2 \mathrm{~d}$ crystals. Mater. Today 2012, 15, 564-589.

(44) Castellanos-Gomez, A.; Vicarelli, L.; Prada, E.; Island, J. O.; Narasimha-Acharya, K. L.; Blanter, S. I.; Groenendijk, D. J.; Buscema, M.; Steele, G. A.; Alvarez, J. V.; Zandbergen, H. W.; Palacios, J. J.; van der Zant, H. S. J., Isolation and characterization of few-layer black phosphorus. 2D Mater. 2014, 1, 025001.

(45) Dresselhaus, M. S.; Dresselhaus, G.; Saito, R.; Jorio, A., Raman spectroscopy of carbon nanotubes. Phys. Rep. 2005, 409, 47-99.

(46) Yang, H.; Fu, B.; Li, D.; Tian, Y.; Chen, Y.; Mattila, M.; Yong, Z.; Li, R.; Hassanien, A.; Yang, C.; Tittonen, I.; Ren, Z.; Bai, J.; Li, Q.; Kauppinen, E. I.; Lipsanen, H.; Sun, Z., Broadband laser polarization control with aligned carbon nanotubes. Nanoscale 2015, 7, 1119911205.

(47) Lee, K. F.; Tian, Y.; Yang, H.; Mustonen, K.; Martinez, A.; Dai, Q.; Kauppinen, E. I.; Malowicki, J.; Kumar, P.; Sun, Z., Photon-Pair Generation with a $100 \mathrm{~nm}$ Thick Carbon Nanotube Film. Adv. Mater. 2017, 1605978.

(48) Y. Akahama, M. K. a. H. K., Raman study of black phosphorus up to 13 GPa. Solid State Commun. 1997, 104, 311-315.

(49) Heyd, J.; Scuseria, G. E.; Ernzerhof, M., Hybrid functionals based on a screened Coulomb potential. J. Chem. Phys. 2003, 118, 8207-8215.

(50) Ares, P.; Zamora, F.; Gomez-Herrero, J., Optical Identification of Few-Layer Antimonene Crystals. ACS Photonics 2017, 4, 600605.

(51) Chen, H.; Fei, W.; Zhou, J.; Miao, C.; Guo, W., Layer Identification of Colorful Black Phosphorus. Small 2017, 13, 1602336.

(52) Low, T.; Rodin, A. S.; Carvalho, A.; Jiang, Y.; Wang, H.; Xia, F.; Castro Neto, A. H., Tunable optical properties of multilayer black phosphorus thin films. Phys. Rev. B 2014, 90, 075434.

(53) Autere, A.; Ryder, C. R.; Saynatjoki, A.; Karvonen, L.; Amirsolaimani, B.; Norwood, R. A.; Peyghambarian, N.; Kieu, K.; Lipsanen, H.; Hersam, M. C.; Sun, Z., Rapid and Large-Area Characterization of Exfoliated Black Phosphorus Using ThirdHarmonic Generation Microscopy. J. Phys. Chem. Lett. 2017, 8, 1343-1350.

(54) Zhong, H.-X.; Gao, S.; Shi, J.-J.; Yang, L., Quasiparticle band gaps, excitonic effects, and anisotropic optical properties of the monolayer distorted1Tdiamond-chain structuresReS $\mathrm{S}_{2}$ and $\mathrm{ReSe}_{2}$. Phys. Rev. B 2015, 92, 115438.

(55) Wen, W.; Zhu, Y.; Liu, X.; Hsu, H. P.; Fei, Z.; Chen, Y.; Wang, X.; Zhang, M.; Lin, K. H.; Huang, F. S.; Wang, Y. P.; Huang, Y. S.; Ho, C. H.; Tan, P. H.; Jin, C.; Xie, L., Anisotropic Spectroscopy and Electrical Properties of 2D $\mathrm{ReS}_{2(1-\mathrm{x})} \mathrm{Se}_{2 x}$ Alloys with Distorted 1T Structure. Small 2017, 13, 1603788.

(56) Elert, G., Refraction, The Physics Hypertextbook. 1998.

(57)https://www.thorlabs.com/newgrouppage9.cfm?objectgrou p_id $=7234$

(58) Tan, D.; Lim, H. E.; Wang, F.; Mohamed, N. B.; Mouri, S.; Zhang, W.; Miyauchi, Y.; Ohfuchi, M.; Matsuda, K., Anisotropic optical and electronic properties of two-dimensional layered germanium sulfide. Nano Res. 2016, 10, 546-555.

(59) Huang, S.; Tatsumi, Y.; Ling, X.; Guo, H.; Wang, Z.; Watson, G.; Puretzky, A. A.; Geohegan, D. B.; Kong, J.; Li, J.; Yang, T.; Saito, R.; Dresselhaus, M. S., In-Plane Optical Anisotropy of Layered Gallium Telluride. ACS Nano 2016, 10, 8964-8972.

(60) Tian, Z.; Guo, C.; Zhao, M.; Li, R.; Xue, J., Two-Dimensional SnS: A Phosphorene Analogue with Strong In-Plane Electronic Anisotropy. ACS Nano 2017, 11, 2219-2226.

(61) Xu, X.; Song, Q.; Wang, H.; Li, P.; Zhang, K.; Wang, Y.; Yuan, K.; Yang, Z.; Ye, Y.; Dai, L., In-Plane Anisotropies of Polarized Raman Response and Electrical Conductivity in Layered Tin Selenide. ACS Appl. Mater. Interfaces 2017, 9, 12601-12607. 


\section{Optical waveplates based on birefringence of anisotropic two-dimensional layered materials}

He Yang, Henri Jussila, Anton Autere, Hannu-Pekka Komsa, Guojun Ye, Xianhui Chen, Tawfique Hasan, and Zhipei Sun

Table of Contents (TOC)

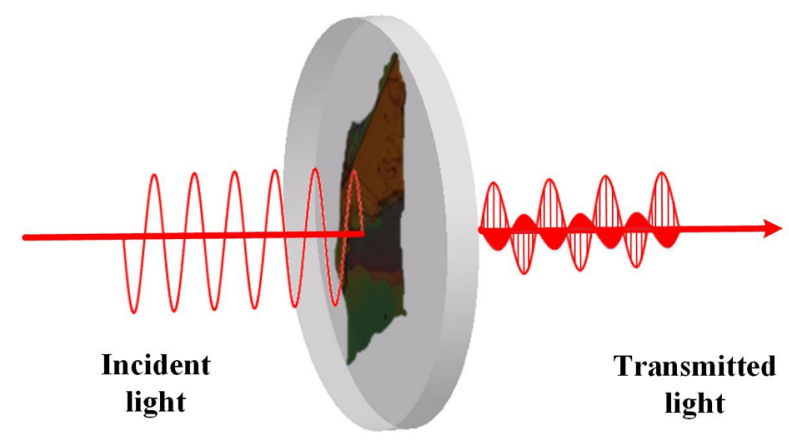

Black

Phosphorus

The polarization state of the light can be changed after it propagates through a black phosphorus flake due to the intrinsic birefringence property of black phosphorus. 